\title{
AVANZADO: REPORTE DE CASOS Y REVISIÓN DE LA LITERATURA
}

\author{
Advanced ectopic abdominal pregnancy: Case \\ report and review of the literature \\ María Fernanda Escobar-Vidarte, $M D^{1}$; Gabriela Caicedo-Herrera, $M^{2}$; \\ Juan David Solarte-Erazo, $M D^{2}$; Laura Sofía Thomas-Pérez, $M D^{2}$; \\ Diana María Dávalos-Pérez, $M D^{3}$; Jaime López-Tenorio, $M^{4}$; \\ Jaime Orrego-Gaviria, $M D^{5}$; Angélica María Forero-Ladino, $M D^{6}$ \\ Recibido: junio 14/16 - Aceptado: marzo 3/17
}

\section{RESUMEN}

Objetivo: reportar dos casos de embarazo ectópico abdominal avanzado, con buen resultado materno/perinatal, y revisar la literatura disponible con respecto al manejo de la placenta y el pronóstico de esta entidad.

Materiales y métodos: se describen dos casos de embarazo ectópico abdominal avanzado en los que se realizó extracción de la placenta, con feto viable y resultado perinatal favorable. Estos fueron atendidos en una institución de cuarto nivel de complejidad,

1 Ginecoobstetra, Universidad del Valle; maestría en Epidemiología Clínica; Universidad de la Frontera, Chile; especialista en Cuidado Intensivo, Universidad del Valle, University of Pittsburgh. Jefe de la Unidad de Alta Complejidad Obstétrica, Fundación Clínica Valle del Lili; coordinadora de la Sección de Cuidado Crítico Obstétrico, Asociación Colombiana de Medicina Crítica y Cuidado Intensivo, Cali (Colombia). mayaev@hotmail.com

2 Médico General, Universidad Icesi, en periodo de servicio social obligatorio, Cali (Colombia).

3 Médico especialista en epidemiologia y salud pública. Jefe del Departamento de Salud Pública, Universidad Icesi, Fundación Valle del Lili, Cali (Colombia).

4 Ginecoobstetra; especialista en Perinatología, Fundación Valle del Lili, Cali (Colombia).

5 Médico pediatra; especialista en Neonatología. Jefe de la Unidad de Neonatología, Fundación Valle del Lili, Cali (Colombia).

6 Médico rural de investigaciones clínicas, Servicio de Ginecología y Obstetricia, Grupo de Investigación en Unidad de Alta Complejidad Obstétrica, Fundación Valle del Lili, Cali (Colombia). ubicada en la ciudad de Cali, Colombia. Se realizó una revisión de la literatura registrada en las bases de datos Medline vía PubMed, con los términos de búsqueda: "embarazo abdominal”, "embarazo esplénico", "embarazo hepático", "embarazo omental” y “embarazo peritoneal”. La búsqueda se limitó a artículos publicados durante los últimos doce años en inglés y español.

Resultados: se recuperaron 228 referencias, de las cuales 42 artículos cumplieron con los criterios de inclusión que informan un total de 74 pacientes con embarazo ectópico abdominal. En cuanto al manejo de la placenta, la remoción de la misma se informó en 42 casos (58\%). La mortalidad materna se presentó en 4 casos $(4,1 \%)$, todos por hemorragia posparto. Un total de 60 pacientes (81\%) presentaron complicaciones, la más frecuente fue hemorragia en 38 de ellas (51\%). En cuanto al resultado perinatal, se encontró una mortalidad perinatal del 43\%.

Conclusión: el manejo de la placenta es el punto clave del que podría depender la frecuencia de complicaciones maternas. Se requieren estudios prospectivos que evalúen el manejo más seguro y efectivo de esta condición.

Palabras clave: embarazo abdominal, embarazo ectópico, hemorragia, resultado del embarazo. 


\section{ABSTRACT}

Objective: Report two cases of advanced ectopic abdominal pregnancy with good maternal/perinatal outcomes, and to review the literature available regarding the management of the placenta and the prognosis for this condition.

Materials and methods: Description of two cases of advanced ectopic abdominal pregnancies with viable fetuses and favourable perinatal outcomes, in which the placenta was removed. The cases were seen at a Level IV institution in the city of Cali, Colombia. Review of the literature registered in the Medline data base via Pubmed using the search terms "abdominal pregnancy", "splenic pregnancy", "hepatic pregnancy", "omental pregnancy" and "peritoneal pregnancy". The search was limited to articles published in English and Spanish during the past 12 years.

Results: Overall, 228 references were retrieved, of which 42 articles reporting on a total of 74 patients with ectopic abdominal pregnancy met the inclusion criteria. Regarding the management of the placenta, it was removed in 42 cases (58\%). There were 4 cases of maternal mortality $(4,1 \%)$ all of them due to postpartum bleeding. Complications occurred in 60 patients (81\%), bleeding being the most frequent in 38 of them (51\%). As for perinatal outcome, perinatal mortality was $43.8 \%$.

Conclusion: Placental management is the the key determinant factor of the frequency of maternal complications. Prospective studies to assess the safest and most effective management of this condition are required.

Key words: Abdominal pregnancy, ectopic pregnancy, bleeding, pregnancy outcome.

\section{INTRODUCCIÓN}

El embarazo ectópico es una condición en la cual el óvulo fecundado es implantado en cualquier sitio diferente de la cavidad uterina (1). El 98\% de los embarazos ectópicos se ubican en la trompa de Falopio. Las implantaciones inusuales suelen presentarse en el cérvix, la porción intersticial de la trompa de
Falopio, el ovario y el abdomen (2). Ocurre en aproximadamente 1,2 al $2 \%$ de los embarazos, con una mortalidad asociada de 0,5 muertes por cada 1000 gestaciones (3).

El embarazo abdominal avanzado es un tipo raro de embarazo ectópico, se define como un embarazo de más de 20 semanas, en el cual el embrión en desarrollo se implanta y crece dentro de la cavidad peritoneal (4). Se clasifica en primario y secundario; el embarazo abdominal primario ocurre cuando el óvulo fertilizado se implanta inicialmente en algún órgano abdominal (menos común) (5), en tanto que el secundario es aquel en el cual la implantación ocurre en la trompa, el ovario o el útero y, posteriormente, escapa a través de una ruptura de la trompa, aborto fimbrial o procedimiento quirúrgico a la cavidad peritoneal $(5,6)$. La incidencia estimada de embarazo abdominal es de 10 por cada 100.000 embarazos (7).

Las manifestaciones clínicas del embarazo ectópico abdominal son múltiples y dependen de la edad gestacional. La sintomatología relatada por la paciente puede variar, desde estar asintomática hasta presentar sintomatología inespecífica o dolor abdominal agudo, lo que hace que el diagnóstico sea complejo de realizar (8). El examen físico abdominal no es muy específico para revelar esta entidad, ya que puede estar enmascarado por otras situaciones como hemoperitoneo, dolor en anexos o masas (9). Frecuentemente es diagnosticado como un embarazo normal o prolongado, que no responde a la inducción y su diagnóstico es intraoperatorio en aproximadamente 50-90\% de los casos (7).

La ecografía es una herramienta útil para el diagnóstico de sospecha de embarazo ectópico (10), detecta presencia o ausencia de saco gestacional dentro o fuera del útero, tiene especificidad (94\%) y sensibilidad (87\%) según un estudio realizado por Condous et al. (11). También se ha descrito el uso de la resonancia magnética nuclear, el problema es que no se encuentra disponible en todas las instituciones de salud y no se tienen datos sobre su exactitud diagnóstica (6). 
La mortalidad asociada al embarazo abdominal ha sido estimada en 5 por cada 1000 casos (7). La causa más común de muerte materna es la separación espontánea de la placenta, que conduce a hemoperitoneo y choque hemorrágico. Además, se acompaña de muerte fetal hasta en un $50 \%$ de los casos (2). El embarazo abdominal también se ha asociado a malformaciones congénitas, cerca del $20 \%$ de los neonatos presentan esta complicación (8). La mortalidad materna varía de acuerdo con el área geográfica, los controles prenatales, la asistencia médica y el estado socioeconómico, y es más frecuente es países en vía de desarrollo (12).

Respecto al abordaje terapéutico, el mayor reto corresponde al manejo de la placenta. Es en esta fase donde se presentan la mayoría de las complicaciones. Se deben tener en cuenta los recursos disponibles en la institución donde se atiende el caso (13).

Dado que es una entidad con incidencia baja, pero con alta tasa de mortalidad materno-fetal, y con marcada variabilidad con respecto al abordaje terapéutico y el pronóstico observado, el objetivo de este estudio es reportar dos casos de embarazo abdominal avanzado y revisar literatura disponible con respecto al tratamiento de la entidad con énfasis en el manejo de la placenta.

\section{PRESENTACIÓN DE LOS CASOS}

Lugar: Fundación Valle del Lili, institución de cuarto nivel de complejidad, centro de referencia médica en el suroccidente colombiano, ubicada en la ciudad de Cali, Colombia, que atiende pacientes pertenecientes al régimen contributivo y subsidiado.

\section{Caso 1}

Paciente de 25 años, G3P1A1, quien ingresa remitida con embarazo de 31,5 semanas de edad gestacional por cuadro clínico de 12 horas de evolución de disnea progresiva y dolor precordial de gran intensidad. Al examen físico se observa tensión arterial (TA): 150/90; frecuencia cardiaca (FC): 89 latidos por minuto (lpm); frecuencia respiratoria (FR): 18 respiraciones por minuto (rpm); frecuencia cardiaca fetal (FCF): 144 lpm; altura uterina: $32 \mathrm{~cm}$, sin actividad uterina; al tacto vaginal se encuentra cérvix completamente desplazado por gran masa que ocupa fondo de saco. Ingresa con sospecha de embarazo abdominal por ecografía realizada a las 23 semanas de edad gestacional, en la que se evidenció alteración de la morfología usual del útero, sin que fuese factible definir los límites de pared uterina alrededor del saco amniótico y sin que fuera evidente la continuidad con el cérvix. Tenía antecedentes, en el embarazo en curso, de laparotomía a la semana 18 de gestación, por torsión de quiste ovárico de $20 \times 20 \mathrm{~cm}$; esta fue realizada en otra institución. En la nota quirúrgica describen que fue imposible de resecar, por lo cual fue drenado; no describen otra alteración. Adicionalmente, a la semana 22 de gestación presentó hidronefrosis bilateral severa atribuible a compresión extrínseca gestacional que mejoró posterior a implantación de dos catéteres doble J. Se inició manejo con sulfato de magnesio y nifedipino con impresión diagnóstica de preeclampsia severa ante la persistencia de cifras tensionales elevadas y epigastralgia en ausencia de lesión de órgano blanco. Se realizó resonancia nuclear magnética (RNM) pélvica que reportó imagen sugestiva de embarazo ectópico abdominal con invasión de la placenta a superficie pélvica. Angiotac pulmonar positivo para tromboembolismo pulmonar (TEP) y ultrasonografía de vías urinarias con hidronefrosis bilateral de predominio izquierdo.

Con el resultado de la RNM y el compromiso clínico de la paciente se tomó la decisión de realizar laparotomía exploratoria en presencia de equipo interdisciplinario conformado por anestesiología, cirugía general, neonatología, cirugía vascular, urología y banco de sangre. En presencia de monitoría invasiva de la madre se realiza laparotomía mediana donde se evidenció embarazo ectópico intraligamentario izquierdo. El útero se encontró ligeramente aumentado de tamaño, con compromiso de su cara posterior por placenta gigante $(30 \times 20 \mathrm{~cm})$ adherida a serosa uterina, asas intestinales y lecho 
vesical. Dada la dificultad del caso, se recurrió a ecografía intraoperatoria para identificar el sitio de incisión para la extracción fetal. Se realizó escisión de cápsula del ligamento para lograr la extracción fetal y se obtuvo un recién nacido vivo con peso de $1205 \mathrm{~g}$, talla $39 \mathrm{~cm}$, puntaje de APGAR 1-3 al 1-5 min, el cual fue trasladado a la unidad de cuidados intensivos neonatales. Posterior a ello, se suturó la cápsula con placenta en su interior, se liberaron adherencias por planos de masa placentaria a útero, vejiga, intestino delgado y colon sigmoide, previa disección de los uréteres en retroperitoneo, los cuales se encontraron severamente dilatados. Se realizó liberación de trompa derecha con pinzamiento, ligadura y corte de pedículos vasculares; una vez liberada la masa se realizó pinzamiento, ligadura y cierre de cúpula sin opción de fijarla a pedículos inferiores por compromiso. Se realizó histerectomía con resección de la masa.

Por presentar sangrado masivo mayor a 3 litros y medio pese al control mecánico, se dejó con empaquetamiento pélvico y drenaje por vacío (vacum pack). Requirió transfusión de 6 unidades de glóbulos rojos, y $1 \mathrm{~g}$ de ácido tranexámico; fue trasladada a la unidad de cuidado intensivo (UCI) para continuar reanimación guiada por metas. En UCI requirió ventilación mecánica invasiva y desarrolló acidosis metabólica con hiperlactatemia, sin requerimiento de vasoactivos; se inició cubrimiento antibiótico con ampicilina-sulbactam y anticoagulación con heparina no fraccionada para el tratamiento de TEP. En las primeras 48 horas de manejo presentó valores de hemoglobina estables, lactato depurado y modulación de respuesta inflamatoria. A las 60 horas se retiró el empaquetamiento, no se evidenció sangrado activo y se realizó cierre definitivo de pared abdominal y extubación programada a las 70 horas. El día 14 de la hospitalización se decide dar egreso con anticoagulación con enoxaparina por 3 a 6 meses. El neonato permaneció por 29 días en la unidad neonatal y no presentó complicaciones mayores. Recibió tratamiento antibiótico por 7 días considerando riesgo de sepsis neonatal.

\section{Caso 2}

Paciente de 30 años, primigestante, con embarazo de 28,3 semanas. Antes del ingreso a la institución presentó cuadro de dolor abdominal intenso asociado a sensación de endurecimiento, por lo cual fue remitida. Ingresó a la unidad de alta complejidad obstétrica con TA: 110/70 mm/Hg; FC: 98 lpm; T: $37^{\circ} \mathrm{C}$; FCF: $130 \mathrm{lpm}$; con dolor severo a la palpación abdominal, sin signos de irritación peritoneal.

Se realizó ecografía obstétrica que arrojó como resultado embarazo ectópico abdominal frente a ruptura uterina. Ultrasonido con limitaciones por dificultades técnicas debido a feto en anhidramnios. Se administraron corticoesteroides para maduración pulmonar fetal y se realizó laparotomía de urgencia, en presencia de equipo interdisciplinario. Se realizó incisión mediana identificando útero placenta percreta y feto en hemiabdomen izquierdo sobre el bazo. Se realizó extracción fetal obteniendo recién nacido vivo, sexo femenino, peso: $1260 \mathrm{~g}$, talla 38 cm, APGAR 7-8, que requirió ventilación por presión positiva y masaje cardiaco, el cual se trasladó a la unidad de cuidados intensivos neonatales (UCIN). Por acretismo placentario que comprometía la cara anterior y posterior uterina, se realizó histerectomía abdominal total. Se trasladó la paciente a UCI, en donde presentó íleo posoperatorio. Al segundo día posoperatorio toleró la vía oral, y su evolución fue favorable, sin evidencia de sangrado, con alta al sexto día de hospitalización.

La recién nacida permaneció hospitalizada por 29 días en UCIN, y luego por 27 días en cuidados intermedios. Durante el periodo neonatal inmediato desarrolló síndrome de dificultad respiratoria aguda (SDRA), y por la sospecha de sepsis neonatal requirió manejo antibiótico. Presentó difícil inicio de nutrición por emesis y distención, los exámenes evidenciaron reflujo gastroesofágico moderado que fue manejado, y se descartó posible malrotación. Los sistemas neurológico, oftalmológico y cardiaco no presentaron ninguna alteración y las posibles anomalías fueron descartadas. 


\section{MATERIALES Y MÉTODOS}

Se llevó a cabo una búsqueda de la literatura en las bases de datos Medline vía PubMed, con los términos de búsqueda: "embarazo abdominal", "embarazo esplénico”, "embarazo hepático”, “embarazo omental” y “embarazo peritoneal”. La búsqueda se limitó a artículos publicados durante los últimos 12 años en inglés y español, que incluían embarazos abdominales $\geq 20$ semanas de gestación. Se incluyeron estudios tipo reporte y series de casos, estudios de cohorte y se descartaron cartas al editor y revisiones de la literatura que estuvieran comprendidas dentro del mismo periodo de tiempo establecido, con la intención de no obtener resultados duplicados. Se excluyeron los estudios en los cuales no hubo acceso al texto completo. Se realizó una selección por título y resumen por dos autores, en caso de encontrar una diferencia se sometió a un tercer evaluador.

Los datos extraídos incluyeron: el número de casos informados, la edad gestacional al momento del diagnóstico, la edad gestacional al parto, el sitio de implantación, el manejo de la placenta, y resultados y complicaciones maternas y fetales.

Aspectos éticos. Se obtuvo consentimiento informado por escrito de las pacientes para la publicación del caso clínico, y se tomaron las precauciones necesarias para garantizar la confidencialidad de la información y el anonimato de las pacientes.

\section{RESULTADOS}

Se recuperaron 228 referencias de las cuales 42 artículos cumplieron con los criterios de inclusión para un total de 74 casos; 38 estudios fueron reportes de casos y 4 series, no se encontró ningún estudio de cohorte. Los resultados de la revisión de la literatura se documentaron en la tabla 1 y se incluyeron los datos de los casos presentados en el presente estudio.

Edad gestacional. La edad gestacional al momento del parto tuvo una mediana de 32 y un rango entre 20 y 43 semanas, en tanto que la edad gestacional al momento del diagnóstico tuvo una mediana de 29 y un rango entre 13 y 43 semanas. En 46 de los

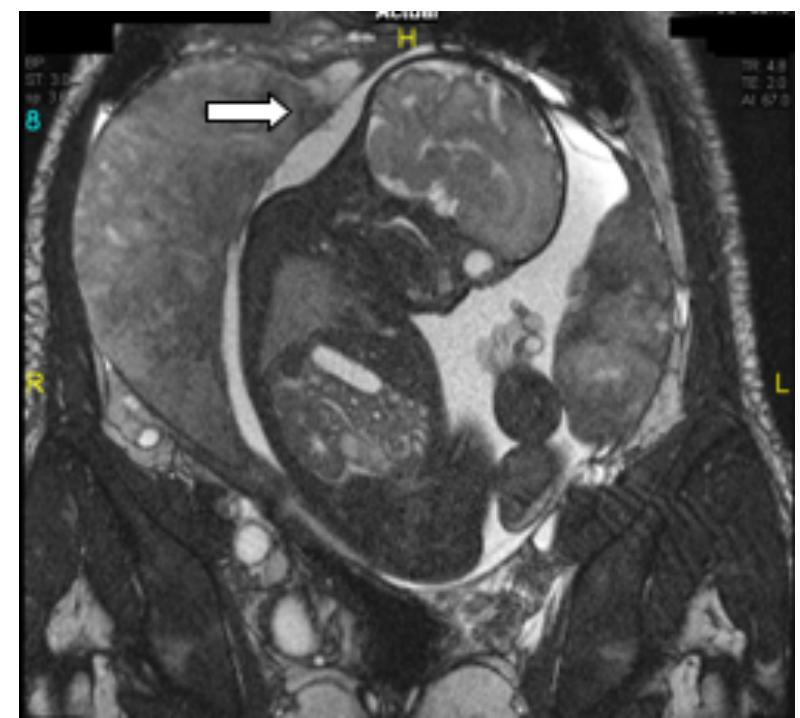

Figura 1. Resonancia magnética de embarazo ectópico abdominal intraligamentario en el cual se observa el feto

casos el diagnóstico de embarazo abdominal fue al momento del parto, en tanto que en 25 el parto se retrasó con manejo médico expectante. El promedio de tiempo entre la edad de diagnóstico y el parto fue de 6,12 semanas.

Placenta. Se describió el sitio de implantación de la placenta en todos los casos reportados, en 37 de ellos la placenta estuvo implantada en un solo lugar, en 5 casos se localizó en la cavidad peritoneal sin que el lugar específico de implantación fuera mencionado. De los 37 casos de implantación única, 14 (37,84\%) se unieron al intestino y $11(32,35 \%)$ al fondo del útero; los sitios de implantación menos frecuentes incluyen omento, 6 (16,22 \%); hígado, 3 (8,11\%); saco de Douglas, 3 (8,11\%), y paredes uterinas o de cavidad abdominal, 2 (5,41 \%) (13, 18, 19, 24, 28, 29, 33, 42, 45, 47, 49, 50, 52, 54). De los 32 casos restantes, 28 presentaron compromiso de dos sitios de implantación y cuatro compromiso de tres o más; de estos, 20 (62,5\%) comprometieron el intestino, 15 (46,88\%) el omento, 8 (25\%) el fondo uterino, 7 (21,88\%) las paredes de cavidad abdominal, 4 $(12,5 \%)$ los anexos, 3 (9,38\%) al saco de Douglas, $2(6,25 \%)$ la vejiga y uno el hígado (13-17, 20-23, 25-27, 30-46, 48, 49, 51, 53). 


\begin{tabular}{|c|c|c|c|c|c|c|c|c|c|}
\hline \multicolumn{10}{|c|}{ Embarazo ectópico abdominal avanzado: revisión sistemática en el periodo 2005 a 2016} \\
\hline$\stackrel{\grave{0}}{\stackrel{2}{2}}$ & 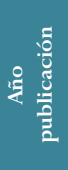 & $\begin{array}{l}\text { है } \\
\text { है } \\
0 \\
\text { हूँ } \\
\text { है } \\
\text { Z }\end{array}$ & 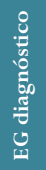 & 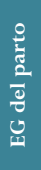 & $\begin{array}{l}\text { Localización } \\
\text { de la placenta }\end{array}$ & $\begin{array}{l}\text { Manejo de la } \\
\text { placenta }\end{array}$ & Resultado materno & Resultado neonatal & Continente \\
\hline \multirow[b]{2}{*}{$\begin{array}{l}\text { Harirah } \\
\text { (14) }\end{array}$} & \multirow[b]{2}{*}{2016} & \multirow[b]{2}{*}{2} & 28 & 29 & $\begin{array}{l}\text { Omento y } \\
\text { pequeña parte } \\
\text { en intestino }\end{array}$ & $\begin{array}{l}\text { Remoción quirúrgica } \\
75 \% \text { que requirió } \\
\text { omentectomía }\end{array}$ & $\begin{array}{l}\text { Vive. Hemorragia } \\
\text { intraperitoneal, transfusión intraoperatoria }\end{array}$ & Vivo, 1225 g. APGAR 6-9 & $\begin{array}{l}\text { América del } \\
\text { Norte (14) }\end{array}$ \\
\hline & & & 23 & 30 & $\begin{array}{l}\text { Fondo del útero, } \\
\text { colon sigmoide y } \\
\text { unión rectosig- } \\
\text { moidal }\end{array}$ & Se deja in situ & $\begin{array}{l}\text { Vive. RNM a los } 6 \text { meses evidencia reduc- } \\
\text { ción de } 1 / 3 \text { de tejido placentario residual }\end{array}$ & $\begin{array}{l}\text { Vivo, } 1085 \text { g, APGAR } 6-8 \text {. } \\
\text { Síndrome por bandas amnió- } \\
\text { ticas en pie derecho }\end{array}$ & \\
\hline $\begin{array}{l}\text { Udigwe } \\
(15)\end{array}$ & 2016 & 1 & 27 & 32 & $\begin{array}{l}\text { Intestino y } \\
\text { anexos derechos }\end{array}$ & $\begin{array}{l}\text { Remoción } \\
\text { quirúrgica }\end{array}$ & $\begin{array}{l}\text { Vive. Sangrado aproximado de } 1 \mathrm{~L} \text {. Recibe } \\
2 \mathrm{U} \text { de sangre. Obstrucción intestinal. } \\
\text { Anexectomía derecha }\end{array}$ & $\begin{array}{l}\text { Vivo, } 2300 \text { g. APGAR 9-10. } \\
\text { Deformidad del pie, asime- } \\
\text { tría facial y clitoromegalia }\end{array}$ & África (15) \\
\hline $\begin{array}{l}\text { Nassali } \\
(16)\end{array}$ & 2016 & 1 & 41 & 41 & $\begin{array}{l}\text { Omento, íleo } \\
\text { mesentérico y se } \\
\text { extiende hasta el } \\
\text { saco de Douglas }\end{array}$ & $\begin{array}{l}\text { Remoción } \\
\text { quirúrgica parcial }\end{array}$ & $\begin{array}{l}\text { Vive. Sangrado mayor a } 2 \mathrm{~L} \text {, hipotensión } \\
\text { intraoperatoria. Requirió de cristaloides, } 2 \\
\text { UGR y } 4 \mathrm{U} \text { de plasma fresco congelado }\end{array}$ & Vivo, 3108 g. APGAR 7-8 & África (16) \\
\hline $\begin{array}{c}\text { Nassali } \\
(17)\end{array}$ & 2016 & 1 & 24 & 28 & $\begin{array}{l}\text { Omento y } \\
\text { fondo } \\
\text { del útero }\end{array}$ & Se deja in situ & $\begin{array}{l}\text { Vive. Sangrado severo, no controlado. } \\
\text { Requirió histerectomía subtotal, transfusión } \\
\text { intraoperatoria de } 2 \text { UGR y } 2 \text { U de plasma }\end{array}$ & $\begin{array}{l}\text { Vivo, } 1500 \mathrm{~g} \text {. APGAR } \\
\text { normal. Dedos de los pies } \\
\text { gangrenados por bandas } \\
\text { omentales a su alrededor }\end{array}$ & África (17) \\
\hline $\begin{array}{l}\text { Bhoil } \\
(18)\end{array}$ & 2016 & 1 & 34 & 34 & $\begin{array}{l}\text { Cavidad peritoneal, } \\
\text { no está bien } \\
\text { especificado }\end{array}$ & $\begin{array}{l}\text { Remoción } \\
\text { quirúrgica }\end{array}$ & Vive & Vivo, 2300 g. APGAR normal & África (18) \\
\hline $\begin{array}{l}\text { Brouard } \\
\text { (19) }\end{array}$ & 2015 & 1 & 37 & 37 & $\begin{array}{l}\text { Lóbulo derecho } \\
\text { hepático } \\
\text { invadiendo } \\
\text { parénquima }\end{array}$ & Se deja in situ & $\begin{array}{l}\text { Vive. Sangrado aproximado de } 1,5 \mathrm{~L} \text {. } \\
\text { Administración de cristaloides y } 2 \mathrm{UGR} .7 \\
\text { semanas RNM evidencia placenta residual } \\
\text { significativa pero asintomática }\end{array}$ & Vivo & África (19) \\
\hline $\begin{array}{l}\text { Mengis- } \\
\operatorname{tu}(20)\end{array}$ & 2015 & 1 & 40 & 40 & $\begin{array}{l}\text { Omento y super- } \\
\text { ficie } \\
\text { serosa del útero }\end{array}$ & $\begin{array}{l}\text { Remoción } \\
\text { quirúrgica }\end{array}$ & $\begin{array}{l}\text { Vive. Sangrado significativo, no controlado } \\
\text { y requiere histerectomía }\end{array}$ & Vivo, $2600 \mathrm{~g}$. & África (20) \\
\hline $\begin{array}{l}\text { Kim } \\
(21)\end{array}$ & 2016 & 1 & 20 & 21 & $\begin{array}{l}\text { Intestino grueso, } \\
\text { omento y anexo } \\
\text { derecho }\end{array}$ & $\begin{array}{l}\text { Remoción } \\
\text { quirúrgica }\end{array}$ & $\begin{array}{l}\text { Vive. Sangrado moderado, adenexotomía } \\
\text { derecha y omentectomía parcial }\end{array}$ & Muerto & \\
\hline \multirow{2}{*}{$\begin{array}{l}\text { Gidiri } \\
(22)\end{array}$} & \multirow[t]{2}{*}{2015} & \multirow[t]{2}{*}{2} & 21 & 23 & $\begin{array}{l}\text { Intestino grueso, } \\
\text { recto y saco de } \\
\text { Douglas }\end{array}$ & $\begin{array}{l}\text { Remoción } \\
\text { quirúrgica }\end{array}$ & Vive. Sangrado aproximado de $1,5 \mathrm{~L}$ & No especificado & $\begin{array}{l}\text { África } \\
(22)\end{array}$ \\
\hline & & & 35 & 35 & $\begin{array}{l}\text { Intestino grueso y } \\
\text { delgado, omento y } \\
\text { fondo del útero }\end{array}$ & Se deja in situ & $\begin{array}{l}\text { Vive. Persistencia de placenta residual hasta } \\
6 \text { meses después de laparotomía }\end{array}$ & Vive, $2000 \mathrm{~g}$ & \\
\hline Lee (23) & 2015 & 1 & NE & 36 & $\begin{array}{l}\text { Colon descenden- } \\
\text { te, riñón izquierdo } \\
\text { y pared abdominal } \\
\text { anterior }\end{array}$ & $\begin{array}{l}\text { Se deja in situ }+ \\
\text { metrotexate }\end{array}$ & $\begin{array}{l}\text { Vive. Empaquetamiento de cavidad } \\
\text { abdominal. } 9 \text { semanas después infección } \\
\text { abdominal y necrosis, requiere control de } \\
\text { daños y } 5 \text { intervenciones quirúrgicas }\end{array}$ & Muerto & África (23) \\
\hline $\begin{array}{l}\text { Bohiltea } \\
\text { (24) }\end{array}$ & 2015 & 1 & 23 & 23 & Fondo del útero & $\begin{array}{l}\text { Remoción } \\
\text { quirúrgica }\end{array}$ & Vive. Sangrado severo & Muerto & \\
\hline $\begin{array}{c}\text { Dabiri } \\
(25)\end{array}$ & 2014 & 1 & 33 & 33 & $\begin{array}{l}\text { Saco de Douglas, } \\
\text { colon sigmoide y } \\
\text { serosa uterina }\end{array}$ & $\begin{array}{l}\text { Remoción } \\
\text { quirúrgica }\end{array}$ & $\begin{array}{l}\text { Vive. Sangrado severo, requirió } 6 \text { UGR, } \\
4 \mathrm{U} \text { de plasma fresco congelado, } 1 \mathrm{U} \text { de } \\
\text { plaquetas }\end{array}$ & Vivo, $2362 \mathrm{~g}$ & $\begin{array}{l}\text { América del } \\
\text { Norte (25) }\end{array}$ \\
\hline $\begin{array}{l}\text { Foue- } \\
\text { lifack } \\
(26)\end{array}$ & 2014 & 1 & 32 & 34 & $\begin{array}{l}\text { Omento, fondo } \\
\text { uterino y asas } \\
\text { intestinales }\end{array}$ & Se deja in situ & $\begin{array}{l}\text { Vive. La placenta y la beta hCG tardaron } \\
\text { once meses en desaparecer por completo }\end{array}$ & Muere, $2600 \mathrm{~g}$. Talipes valgo & $\begin{array}{l}\text { África } \\
(26)\end{array}$ \\
\hline $\begin{array}{l}\text { Huang } \\
(27)\end{array}$ & 2014 & 1 & 26 & 33 & $\begin{array}{l}\text { Colon sigmoide } \\
\text { y recto }\end{array}$ & $\begin{array}{l}\text { Se deja in situ }+ \\
\text { mifepristone y } \\
\text { metrotexate }\end{array}$ & Vive. Embarazo heterotópico & Muerto, $1600 \mathrm{~g}$ & \\
\hline $\begin{array}{l}\text { Marcellin } \\
\text { (28) }\end{array}$ & 2014 & 1 & 22 & 32 & Omento & Se deja in situ & $\begin{array}{l}\text { Vive. Sangrado moderado, cateterismo y } \\
\text { embolización arterial selectiva }\end{array}$ & $\begin{array}{l}\text { Vivo, } 2300 \text { g. Requirió IOT } \\
\text { por } 6 \text { horas }\end{array}$ & $\begin{array}{l}\text { Europa } \\
(28)\end{array}$ \\
\hline $\begin{array}{l}\text { Joshi } \\
(29)\end{array}$ & 2014 & 1 & 34 & 34 & Intestino & $\begin{array}{l}\text { Remoción } \\
\text { quirúrgica }\end{array}$ & $\begin{array}{l}\text { Vive. Sangrado aproximado de 1,3L. } \\
\text { Requirió } 4 \mathrm{UGR}\end{array}$ & $\begin{array}{l}\text { Vivo. Asfixia perinatal y } \\
\text { malformaciones congéni- } \\
\text { tas NE }\end{array}$ & $\begin{array}{l}\text { Asia } \\
(29)\end{array}$ \\
\hline
\end{tabular}




\begin{tabular}{|c|c|c|c|c|c|c|c|c|c|}
\hline & & & Eml & $\mathrm{raz}$ & ectópico abdo & ninal avanzado: & $\begin{array}{l}\text { bla } 1 \\
\text { evisión sistemática en el period }\end{array}$ & o 2005 a 2016 & \\
\hline$\stackrel{\grave{2}}{\stackrel{2}{z}}$ & 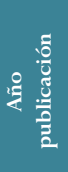 & $\begin{array}{l}\text { है } \\
\text { है } \\
\text { ¿ू } \\
\vdots \\
\text { हू } \\
\text { Z }\end{array}$ & 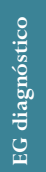 & 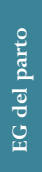 & $\begin{array}{l}\text { Localización } \\
\text { de la placenta }\end{array}$ & $\begin{array}{l}\text { Manejo de la } \\
\text { placenta }\end{array}$ & Resultado materno & Resultado neonatal & Continente \\
\hline $\begin{array}{l}\text { Mato- } \\
\text { velo- } \\
(30)\end{array}$ & 2014 & 1 & 31 & 32 & $\begin{array}{l}\text { Omento y cuerno } \\
\text { derecho }\end{array}$ & $\begin{array}{l}\text { Remoción } \\
\text { quirúrgica }\end{array}$ & Vive. Hemoperitoneo y anemia severa & Vivo, 1700 g. APGAR 8-10 & \\
\hline $\begin{array}{l}\text { Renfroe } \\
\text { (31) }\end{array}$ & 2013 & 1 & 20 & 24 & $\begin{array}{l}\text { Colon sigmoide y } \\
\text { fondo del útero }\end{array}$ & $\begin{array}{l}\text { Remoción } \\
\text { quirúrgica }\end{array}$ & $\begin{array}{l}\text { Vive. Sangrado severo aproximadamente } \\
5,5 \mathrm{~L}, 12 \mathrm{UGR}, 10 \mathrm{U} \text { de plasma fresco con- } \\
\text { gelado, } 4 \mathrm{U} \text { plaquetas y } 1 \mathrm{U} \text { crioprecipitado }\end{array}$ & $\begin{array}{l}\text { Vivo, } 660 \text { g. APGAR 2-4-5. } \\
\text { Displasia broncopulmonar }\end{array}$ & \\
\hline $\begin{array}{c}\text { Smrtka } \\
(32)\end{array}$ & 2013 & 1 & 23 & 23 & $\begin{array}{l}\text { Colon recto } \\
\text { sigmoide y ovario } \\
\text { izquierdo }\end{array}$ & $\begin{array}{l}\text { Remoción } \\
\text { quirúrgica }\end{array}$ & $\begin{array}{l}\text { Vive. Sangrado mínimo } 600 \mathrm{~mL} \text {, } \\
\text { ooforectomía izquierda }\end{array}$ & $\begin{array}{l}\text { Vivo, } 580 \text { g. APGAR 5-7. } \\
\text { Hospitalización en UCI } \\
\text { por } 5 \text { meses }\end{array}$ & $\begin{array}{l}\text { América del } \\
\text { Norte (32) }\end{array}$ \\
\hline \multirow{6}{*}{$\begin{array}{l}\text { Aliyu } \\
\text { (33) }\end{array}$} & \multirow{6}{*}{2013} & \multirow{6}{*}{6} & 32 & 32 & Fondo del útero & $\begin{array}{l}\text { Remoción } \\
\text { quirúrgica }\end{array}$ & $\begin{array}{l}\text { Vive. Sepsis de origen } \\
\text { obstétrico }\end{array}$ & Muerte intraabdominal & África (33) \\
\hline & & & 42 & 42 & Intestino & $\begin{array}{l}\text { Se deja in situ. Remo- } \\
\text { ción en } 2 \text { da cirugía }\end{array}$ & $\begin{array}{l}\text { Vive. Obstrucción intestinal e infección } \\
\text { después de } 5 \text { semanas, requirió remoción } \\
\text { de tejido placentario necrótico }\end{array}$ & Vivo, 3200 g. APGAR 5-7 & \\
\hline & & & 43 & 43 & $\begin{array}{l}\text { Omento y tubo } \\
\text { izquierdo }\end{array}$ & $\begin{array}{l}\text { Remoción } \\
\text { quirúrgica }\end{array}$ & Vive & $\begin{array}{l}\text { Muerto, } 2400 \mathrm{~g} \text {. Macerado, } \\
\text { malformaciones craneales y } \\
\text { miembros }\end{array}$ & \\
\hline & & & 35 & 35 & Intestino y omento & $\begin{array}{l}\text { Se deja in situ. Remo- } \\
\text { ción en } 2 \text { da cirugía }\end{array}$ & $\begin{array}{l}\text { Vive. Obstrucción intestinal e infección } \\
\text { después de } 4 \text { semanas, requirió remoción } \\
\text { de tejido placentario necrótico }\end{array}$ & $\begin{array}{l}\text { Vivo, } 3200 \text { g. APGAR } 4-6 \text {. } \\
\text { Muerte posterior a las } 24 \\
\text { horas }\end{array}$ & \\
\hline & & & 39 & 39 & $\begin{array}{l}\text { Cavidad peritoneal, } \\
\text { no está bien } \\
\text { especificado }\end{array}$ & $\begin{array}{l}\text { Remoción } \\
\text { quirúrgica }\end{array}$ & Muerte. Sangrado severo no controlado & Muerto y macerado & \\
\hline & & & 33 & 33 & Omento & $\begin{array}{l}\text { Remoción } \\
\text { quirúrgica }\end{array}$ & $\begin{array}{l}\text { Vive. Sangrado leve, } \\
\text { requiere } 2 \text { UGR } \\
\text { intraoperatoriamente }\end{array}$ & Muerto, $1500 \mathrm{~g}$, macerado & \\
\hline \multirow[b]{2}{*}{$\begin{array}{l}\text { Mpogoro } \\
\text { (34) }\end{array}$} & \multirow[b]{2}{*}{2013} & \multirow[b]{2}{*}{2} & \multirow[b]{2}{*}{26} & \multirow[b]{2}{*}{26} & $\begin{array}{l}\text { Ciego y colon } \\
\text { ascendente }\end{array}$ & \multirow{2}{*}{$\begin{array}{l}\text { Se deja in situ. } \\
\text { Remoción en } \\
\text { 2da cirugía }\end{array}$} & \multirow{2}{*}{$\begin{array}{l}\text { Vive. Sangrado aproximado de } 2 \mathrm{~L} \text {. Requirió } \\
\text { de } 4 \text { UGR. } 14 \text { dias POP peritonitis, lavado. } \\
\text { Remoción de tejido placentario con sangra- } \\
\text { do severo, empaquetamiento por } 2 \text { días }\end{array}$} & Muerto, $700 \mathrm{~g}$ & \multirow[b]{2}{*}{ África (34) } \\
\hline & & & & & $\begin{array}{l}\text { Colon transverso } \\
\text { y sigmoide }\end{array}$ & & & Muerto, $800 \mathrm{~g}$ & \\
\hline $\begin{array}{l}\text { Pradhan } \\
\text { (35) }\end{array}$ & 2013 & 1 & 26 & 26 & $\begin{array}{l}\text { Mesocolon, colon } \\
\text { ascendente y pared } \\
\text { medial del ciego }\end{array}$ & $\begin{array}{l}\text { Hallazgos } \\
\text { post mortem }\end{array}$ & $\begin{array}{l}\text { Muerte. Acretismo placentario, hemorragia } \\
\text { masiva }\end{array}$ & Muerto, $985 \mathrm{~g}$ & Asia (35) \\
\hline $\begin{array}{l}\text { Masuku- } \\
\text { me (36) }\end{array}$ & 2013 & 1 & 40 & 40 & $\begin{array}{l}\text { Intestino grueso } \\
\text { y delgado, parte } \\
\text { superior de la } \\
\text { vejiga }\end{array}$ & $\begin{array}{l}\text { Remoción } \\
\text { quirúrgica }\end{array}$ & $\begin{array}{l}\text { Vive. Sangrado aproximado de 1,5L. } \\
\text { Requirió } 3 \text { UGR. Ascitis }\end{array}$ & Vivo, $2850 \mathrm{~g}$, APGAR 8-10 & África (36) \\
\hline Kim (37) & 2013 & 1 & 18 & 34 & $\begin{array}{l}\text { Colon sigmoide } \\
\text { y pared uterina } \\
\text { posterior }\end{array}$ & $\begin{array}{l}\text { Remoción } \\
\text { quirúrgica }\end{array}$ & $\begin{array}{l}\text { Vive. Sangrado moderado, requirió } 4 \text { UGR, } \\
2 \text { U plasma fresco } \\
\text { congelado y } 10 \mathrm{U} \text { de plaquetas }\end{array}$ & Vivo, 2100 g, APGAR 5-8 & Asia (37) \\
\hline $\begin{array}{c}\text { Mittal } \\
(38)\end{array}$ & 2012 & 1 & 26 & 26 & $\begin{array}{l}\text { Superficie perito- } \\
\text { neal de la pared } \\
\text { abdominal anterior }\end{array}$ & Se deja in situ & $\begin{array}{l}\text { Vive. Múltiples lavados peritoneales, } \\
\text { antibiótico y transfusión sanguínea }\end{array}$ & Muerto, macerado & Asia (38) \\
\hline $\begin{array}{l}\text { Collins } \\
(39)\end{array}$ & 2011 & 1 & 27 & 30 & $\begin{array}{l}\text { Cara lateral } \\
\text { derecha del piso } \\
\text { pélvico }\end{array}$ & $\begin{array}{l}\text { Remoción } \\
\text { quirúrgica }\end{array}$ & Vive & Vivo & Europa (39) \\
\hline $\begin{array}{c}\text { Dahab } \\
(40)\end{array}$ & 2011 & 1 & 13 & 40 & $\begin{array}{l}\text { Cara posterior del } \\
\text { ligamento ancho } \\
\text { izquierdo }\end{array}$ & $\begin{array}{l}\text { Remoción } \\
\text { quirúrgica }\end{array}$ & $\begin{array}{l}\text { Vive. Sangrado severo, transfusión masiva. } \\
7 \text { UGR, } 4 \text { U plasma fresco congelado, } 10 \mathrm{U} \\
\text { crioprecipitado }\end{array}$ & Vivo, APGAR 6-10 & Asia (40) \\
\hline $\begin{array}{c}\text { Baffoe } \\
(41)\end{array}$ & 2011 & 1 & 38 & 38 & $\begin{array}{l}\text { Intestino grueso, } \\
\text { omento y región } \\
\text { izquierda del útero }\end{array}$ & $\begin{array}{l}\text { Remoción } \\
\text { quirúrgica }\end{array}$ & $\begin{array}{l}\text { Vive. Sangrado aproximado } 1 \mathrm{~L} \text {, transfusión } \\
1 \mathrm{U} \text { sangre }\end{array}$ & Vivo, $2300 \mathrm{~g}$ & \\
\hline $\begin{array}{c}\text { Brewster } \\
(42)\end{array}$ & 2011 & 1 & 20 & 33 & $\begin{array}{l}\text { Pared posterior del } \\
\text { útero }\end{array}$ & $\begin{array}{l}\text { Remoción } \\
\text { quirúrgica }\end{array}$ & Vive & Vivo & Asia (42) \\
\hline
\end{tabular}




\begin{tabular}{|c|c|c|c|c|c|c|c|c|c|}
\hline & & & & & & & abla 1 & & \\
\hline & & & Eml & $\operatorname{araz}$ & ectópico abdo & minal avanzado: & evisión sistemática en el perioc & o 2005 a 2016 & \\
\hline$\stackrel{\grave{3}}{\frac{3}{2}}$ & 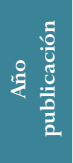 & 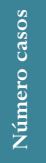 & 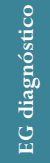 & 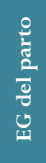 & $\begin{array}{l}\text { Localización } \\
\text { de la placenta }\end{array}$ & $\begin{array}{l}\text { Manejo de la } \\
\text { placenta }\end{array}$ & Resultado materno & Resultado neonatal & Continente \\
\hline $\begin{array}{l}\text { Ramphal } \\
(43)\end{array}$ & 2010 & 1 & 19 & 34 & $\begin{array}{l}\text { Bajo la superficie } \\
\text { del lóbulo derecho } \\
\text { del hígado }\end{array}$ & $\begin{array}{l}\text { Se deja in situ }+ \\
\text { metotexate }\end{array}$ & Vive & Vivo & África (43) \\
\hline $\begin{array}{l}\text { Gupta } \\
(44)\end{array}$ & 2009 & 1 & 32 & 41 & $\begin{array}{l}\text { Omento e } \\
\text { intestinos }\end{array}$ & $\begin{array}{l}\text { Remoción } \\
\text { quirúrgica }\end{array}$ & $\begin{array}{l}\text { Vive. Sangrado severo, } \\
\text { requiere transfusión } 7 \text { U sangre }\end{array}$ & Vivo & Asia (44) \\
\hline & & & 25 & 25 & Hígado & Se deja in situ & Vive & Muerto, $650 \mathrm{~g}$ & África (13) \\
\hline & & & 37 & 37 & Omento e intestino & $\begin{array}{l}\text { Se deja in situ }+ \text { me- } \\
\text { totrexate. Remoción } \\
\text { en } 2 \text { da cirugía }\end{array}$ & $\begin{array}{l}\text { Vive. } 4 \text { meses POP dolor } \\
\text { abdominal severo, requiere } 2 \mathrm{da} \\
\text { laparotomía }\end{array}$ & Vivo, 2950 g. APGAR 9-10 & \\
\hline & & & 33 & 33 & Intestino & Se deja in situ & Vive & Vivo, 2600 g. APGAR 8-10 & \\
\hline $\begin{array}{l}\text { Oneko } \\
(13)\end{array}$ & 2009 & 7 & 20 & 20 & Intestino & $\begin{array}{l}\text { Se deja in situ }+ \\
\text { metrotexate }\end{array}$ & $\begin{array}{l}\text { Vive. Anemia severa, } \\
\text { transfusión de } 4 \text { UGR }\end{array}$ & Muerto, $450 \mathrm{~g}$ & \\
\hline & & & 20 & 20 & Intestino & $\begin{array}{l}\text { Remoción } \\
\text { quirúrgica }\end{array}$ & $\begin{array}{l}\text { Vive. Sangrado, empaquetamiento } \\
\text { abdominal }\end{array}$ & Muerto, $430 \mathrm{~g}$ & \\
\hline & & & 38 & 38 & Intestino & $\begin{array}{l}\text { Remoción } \\
\text { quirúrgica }\end{array}$ & $\begin{array}{l}\text { Vive. Hemoperitoneo, } \\
\text { transfusión } 4 \text { UGR }\end{array}$ & Muerto, $2400 \mathrm{~g}$ & \\
\hline & & & 22 & 22 & $\begin{array}{l}\text { Intraabdominal, } \\
\text { No especificado }\end{array}$ & $\begin{array}{l}\text { Remoción } \\
\text { quirúrgica }\end{array}$ & $\begin{array}{l}\text { Vive. Hemoperitoneo, } \\
\text { transfusión 3UGR }\end{array}$ & Muerto, $500 \mathrm{~g}$ & \\
\hline & & & NE & $\mathrm{NE}$ & Intestino & $\begin{array}{l}\text { Remoción } \\
\text { quirúrgica }\end{array}$ & Vive, transfusión sanguínea & Muerte, macerado, $2250 \mathrm{~g}$ & África (45) \\
\hline & & & 36 & 36 & Omento & $\begin{array}{l}\text { Hallazgos post } \\
\text { mortem }\end{array}$ & $\begin{array}{l}\text { Muerte. Colapso cardiovascular y hemo- } \\
\text { peritoneo }\end{array}$ & Muerto & \\
\hline $\begin{array}{l}\text { Sunday- } \\
\text { adeoye }\end{array}$ & 2009 & 10 & 37 & 37 & Fondo uterino & $\begin{array}{l}\text { Remoción } \\
\text { quirúrgica }\end{array}$ & Vive & Vivo, $2650 \mathrm{~g}$ & \\
\hline (45) & & & 33 & 33 & $\begin{array}{l}\text { Pared abdominal } \\
\text { posterior }\end{array}$ & Se deja in situ & Vive. Transfusión sanguínea & Muerto, macerado, $1500 \mathrm{~g}$ & \\
\hline & & & 28 & 28 & Omento & $\begin{array}{l}\text { Remoción } \\
\text { quirúrgica }\end{array}$ & Vive. Transfusión sanguínea & Muerto, macerado, $800 \mathrm{~g}$ & \\
\hline & & & 33 & 33 & Fondo uterino & $\begin{array}{l}\text { Remoción } \\
\text { quirúrgica }\end{array}$ & Vive. Transfusión sanguínea & Muerto, macerado, $2800 \mathrm{~g}$ & \\
\hline & & & 38 & 38 & Fondo uterino & $\begin{array}{l}\text { Remoción } \\
\text { quirúrgica }\end{array}$ & Vive & Muerto, macerado $2100 \mathrm{~g}$ & \\
\hline & & & 28 & 28 & Omento & Se deja in situ & Vive & Muerto, macerado $600 \mathrm{~g}$ & \\
\hline & & & 34 & 34 & Saco de douglas & $\begin{array}{l}\text { Remoción } \\
\text { quirúrgica }\end{array}$ & Vive & Muerto, $1700 \mathrm{~g}$ & \\
\hline & & & 29 & 29 & $\begin{array}{l}\text { Fondo uterino e } \\
\text { intestino }\end{array}$ & $\begin{array}{l}\text { Remoción } \\
\text { quirúrgica }\end{array}$ & Vive & Muerto, $700 \mathrm{~g}$ & \\
\hline & & & 30 & 30 & $\begin{array}{l}\text { Pared pélvica y } \\
\text { vejiga }\end{array}$ & Se deja in situ & $\begin{array}{l}\text { Vive. Transfusión 3UGR. Abceso de placen- } \\
\text { ta } 10 \text { días POP, manejo médico }\end{array}$ & Vivo, RCIU & Asia (46) \\
\hline (46) & & & 23 & 23 & $\begin{array}{l}\text { Colon sigmoide } \\
\text { y pared pélvica } \\
\text { derecha }\end{array}$ & $\begin{array}{l}\text { Se deja in situ. } \\
\text { Remoción en 2da } \\
\text { cirugía }\end{array}$ & $\begin{array}{l}\text { Vive. Abceso placentario } 2 \text { días POP, segun- } \\
\text { da laparotomía e } \\
\text { infección del sitio quirúrgico }\end{array}$ & Muerto, macerado & \\
\hline $\begin{array}{l}\text { Isah } \\
(47)\end{array}$ & 2008 & 1 & 39 & 39 & Fondo uterino & $\begin{array}{l}\text { Remoción } \\
\text { quirúrgica }\end{array}$ & Vive. Sangrado 0,9L, transfusión de 2 UGR & Vivo, $3200 \mathrm{~g}$. APGAR 7-8 & $\begin{array}{l}\text { África } \\
(47)\end{array}$ \\
\hline $\begin{array}{c}\text { Promsonthi } \\
(48)\end{array}$ & 2007 & 1 & 40 & 40 & $\begin{array}{l}\text { Pared peritoneal } \\
\text { posterior y fondo } \\
\text { del útero }\end{array}$ & $\begin{array}{l}\text { Se deja in situ. } \\
\text { Remoción en } 2 \text { da } \\
\text { cirugía }\end{array}$ & $\begin{array}{l}\text { Vive. Sangrado severo intraoperatorio. } \\
\text { Abceso abdominal } 3 \text { meses después de } \\
\text { laparotomía }\end{array}$ & Vivo, $3540 \mathrm{~g}$ & $\begin{array}{c}\text { Europa } \\
(48)\end{array}$ \\
\hline
\end{tabular}




\begin{tabular}{|c|c|c|c|c|c|c|c|c|c|}
\hline & & & Eml & raz & ectópico abd & minal avanza & $\begin{array}{l}\text { abla } 1 \\
\text { revisión sistemática en el }\end{array}$ & 2005 a 2016 & \\
\hline 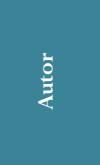 & 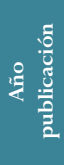 & 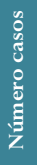 & 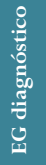 & 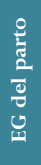 & $\begin{array}{l}\text { Localización } \\
\text { de la placenta }\end{array}$ & $\begin{array}{c}\text { Manejo de la } \\
\text { placenta }\end{array}$ & Resultado materno & Resultado neonatal & Continente \\
\hline \multirow{9}{*}{$\begin{array}{l}\text { Lockhat } \\
\text { (49) }\end{array}$} & \multirow{9}{*}{2006} & \multirow{9}{*}{9} & 28 & 28 & $\begin{array}{l}\text { Fondo del útero } \\
\text { lado derecho }\end{array}$ & $\begin{array}{l}\text { Remoción } \\
\text { quirúrgica }\end{array}$ & Vive & Vivo, $1500 \mathrm{~g}$ & $\begin{array}{c}\text { África } \\
(49)\end{array}$ \\
\hline & & & 26 & 29 & Bolsa de Douglas & $\begin{array}{l}\text { Remoción } \\
\text { quirúrgica }\end{array}$ & Vive & Muerto & \\
\hline & & & 23 & 31 & Hígado & Se deja in situ & Vive. Placenta retenida & Vivo, $1400 \mathrm{~g}$ & \\
\hline & & & 29 & 29 & $\begin{array}{l}\text { Fondo del útero } \\
\text { lado derecho }\end{array}$ & $\begin{array}{l}\text { Remoción } \\
\text { quirúrgica }\end{array}$ & Vive & Vivo & \\
\hline & & & 25 & 31 & $\begin{array}{l}\text { Ligamento pélvico } \\
\text { derecho y omento }\end{array}$ & $\begin{array}{l}\text { Remoción } \\
\text { quirúrgica }\end{array}$ & Vive & Vivo, $1800 \mathrm{~g}$ & \\
\hline & & & 20 & 21 & Fondo del útero & Se deja in situ & Vive. Placenta retenida & $\begin{array}{l}\text { Muerto. Anomalías con- } \\
\text { génitas }\end{array}$ & \\
\hline & & & 20 & 21 & Bolsa de Douglas & Se deja in situ & Vive. Placenta retenida, eclampsia & Muerto & \\
\hline & & & 30 & 31 & $\begin{array}{l}\text { Fondo del útero } \\
\text { lado derecho y } \\
\text { omento }\end{array}$ & $\begin{array}{l}\text { Remoción } \\
\text { quirúrgica }\end{array}$ & Vive & Vivo, $1500 \mathrm{~g}$ & \\
\hline & & & 28 & 31 & Fondo del útero & $\begin{array}{l}\text { Remoción } \\
\text { quirúrgica }\end{array}$ & Vive & Vivo, $1500 \mathrm{~g}$ & \\
\hline $\begin{array}{l}\text { Roberts } \\
(50)\end{array}$ & 2006 & 1 & 35 & 35 & Peritoneo & $\begin{array}{l}\text { Se deja in situ. } \\
\text { Remoción en } 2 \text { da } \\
\text { cirugía }\end{array}$ & Vive. Hidronefrosis y ascitis & Vivo & $\begin{array}{l}\text { Australia } \\
\quad(50)\end{array}$ \\
\hline $\begin{array}{l}\text { Tshivhula } \\
\text { (51) }\end{array}$ & 2006 & 1 & 29 & 32 & $\begin{array}{l}\text { Fondo uterino y } \\
\text { ligamento ancho } \\
\text { derecho. Colon e } \\
\text { intestino delgado }\end{array}$ & $\begin{array}{l}\text { Remoción } \\
\text { quirúrgica }\end{array}$ & Vive. Picos febriles & $\begin{array}{l}\text { Vivo, } 1416 \text { g. APGAR } 10 . \\
\text { Enfermedad de membrana } \\
\text { hialina }\end{array}$ & África (51) \\
\hline $\begin{array}{l}\text { Godyn } \\
(52)\end{array}$ & 2006 & 1 & NR & 37 & $\begin{array}{l}\text { Cavidad peritoneal, } \\
\text { no está bien } \\
\text { especificado }\end{array}$ & $\begin{array}{l}\text { Remoción } \\
\text { quirúrgica }\end{array}$ & NR & Vivo, $3255 \mathrm{~g}$ & $\begin{array}{l}\text { América del } \\
\text { Norte (52) }\end{array}$ \\
\hline $\begin{array}{l}\text { Cetinkaya } \\
\text { (53) }\end{array}$ & 2005 & 1 & 36 & 36 & $\begin{array}{l}\text { Intestino delgado } \\
\text { y omento }\end{array}$ & Se deja in situ & $\begin{array}{l}\text { Vive. Hospitalización } \\
\text { prolongada por } 5 \text { semanas }\end{array}$ & Muerto & $\begin{array}{l}\text { Asia-Europa } \\
\quad(53)\end{array}$ \\
\hline $\begin{array}{l}\text { Xiao } \\
(54)\end{array}$ & 2005 & 1 & 31 & 31 & Omento & $\begin{array}{l}\text { Se deja in situ }+ \\
\text { Metrotexate }\end{array}$ & Vive & Vivo, 1745 g. APGAR 5-8-10 & Asia (54) \\
\hline \multirow{2}{*}{$\begin{array}{l}\text { Nuestros } \\
\text { casos }\end{array}$} & \multirow{2}{*}{2016} & \multirow{2}{*}{2} & 32 & 32 & $\begin{array}{l}\text { Cara posterior } \\
\text { del útero, intestino, } \\
\text { vejiga }\end{array}$ & $\begin{array}{l}\text { Remoción } \\
\text { quirúrgica }\end{array}$ & $\begin{array}{l}\text { Vive. Histerectomía abdominal. Ileo } \\
\text { posquirúrgico }\end{array}$ & Vivo, 1205 g. APGAR 1-3 & Sur América \\
\hline & & & 28 & 28 & $\begin{array}{l}\text { Cara anterior y } \\
\text { posterior del útero }\end{array}$ & $\begin{array}{l}\text { Remoción } \\
\text { quirúrgica }\end{array}$ & Vive & $\begin{array}{l}\text { Vivo, } 1260 \text { g. APGAR 7-8-9. } \\
\text { Síndrome de dificultad } \\
\text { respiratoria }\end{array}$ & Sur América \\
\hline
\end{tabular}

El manejo de la placenta se describió en 72 casos. En 29 casos la placenta se dejó in situ $(13,14,17$, 19, 22, 23, 26-28, 33, 34, 38, 43, 45, 46, 48-50, 53, 54). De esos 29,5 recibieron metrotexate $(13,23$, 43, 54), 6 requirieron remoción quirúrgica durante una segunda intervención $(13,33,46,48,50)$ y uno recibió los dos manejos (27). La placenta fue removida quirúrgicamente en 42 casos y de estos, dos requirieron histerectomía $(17,20)$ o salpingooforectomía unilateral $(15,21)$, siendo la hemorragia la principal complicación (13-16, 18, 20-22, 24, 25, 29-33, 36, 37, 39-42, 44, 45, 47, 49, 51, 52). En dos de los casos no se mencionó el manejo de la placenta dado que se trató de un hallazgo post mortem $(35,45)$.

Resultados maternos. Los resultados maternos se documentaron en 73 de los casos. Ocurrieron 
tres $(4,11 \%)$ muertes maternas siendo la causa la hemorragia masiva $(33,35,45)$. En 60 de los casos se reportaron complicaciones maternas, siendo las más comunes el sangrado (moderado a severo) en 38 (63,33\%) de los casos y, consecuentemente, el requerimiento de transfusiones sanguíneas en 30 (50\%) de ellos, estas se presentaron en su mayoría en la remoción de la placenta (13-17, 19-25, 28-31, 33-38, 40, 41, 44-48). Otras complicaciones menos frecuentes fueron la infección, 11 (18,33\%) (23, $33,34,38,46,48,51)$; la necesidad de cirugía adicional, 9 (15\%) (13, 14, 17, 20, 21, 23, 28, 34, 38); el requerimiento de empaquetamiento o control de daños, 4 (6,67\%) (13, 23, 34); la obstrucción intestinal, $3(5 \%)(15,33)$; la placenta retenida, 3 (5\%) (49) y, finalmente, la ascitis, 2 (3,33\%) (36, 50). Es importante aclarar que estas complicaciones no son excluyentes entre sí, y una misma paciente podía presentar más de una.

Resultados perinatales. Se describieron los resultados perinatales en 73 de los casos; 41 (56,16\%) de ellos sobrevivieron (13-20, 22, 25, 28-33, 36, 37, 39, 44$52,54)$, en tanto que $32(43,84 \%)$ fueron mortinatos o muertes neonatales tempranas $(13,21,23,24,26$, 27, 33-35, 38, 45, 46, 49, 53). El peso al nacer fue descrito en 51 de los casos con un promedio $1841 \pm$ 882,63 g. Dos de los recién nacidos sufrieron deformaciones de las extremidades por bandas amnióticas y omentales $(14,17)$. Se identificaron cinco casos de anomalías congénitas o características dismórficas (talipes, asimetría facial y contracturas de extremidades), de los cuales tres murieron y uno cursó con restricción de crecimiento intrauterino (RCIU) severo $(15,26,29,31,33,49)$. Las puntuaciones de APGAR se documentaron en 17 casos con un promedio de 6,5 al primer minuto y 8,3 a los 5 minutos $(13-18,30-33,36,37,40,47,51,54)$.

\section{CONCLUSIONES}

El embarazo ectópico abdominal es una condición de alto riesgo para la madre y el feto. Se acompaña de una importante morbimortalidad materna y alta mortalidad perinatal. El manejo de la placenta es el punto clave del que podría depender la frecuencia de complicaciones maternas. Se requieren estudios prospectivos, idealmente aleatorizados, que evalúen el manejo más seguro y efectivo de la placenta en esta condición.

\section{REFERENCIAS}

1. The American College of Obstetrics and Gynecologist. Ectopic pregnancy. 2011 [visitado 2017 Feb 15]. Disponible en: https://www.acog.org/-/media/ For-Patients/faq155.pdf?dmc $=1 \&$ ts $=2017012$ 5T1456405281.

2. Nkusu Nunyalulendho D, Einterz EM. Advanced abdominal pregnancy: Case report and review of 163 cases reported since 1946. Rural Remote Health. 2008;8:1087.

3. Atrash HK, Friede A, Hogue CJ. Abdominal pregnancy in the United States: Frequency and maternal mortality. Obstet Gynecol. 1987;69:333-7.

4. Costa SD, Presley J, Bastert G. Advanced abdominal pregnancy. Obstet Gynecol Surv. 1991;46:515-25.

5. Okafor I, Ude A, Aderibigbe ASO, Amu O, Udeh P, Obianyo N, et al. Abdominal pregnancy-a case report. J West Afr Coll Surg. 2011;1:121-30.

6. Martin JN Jr, Sessums JK, Martin RW, Pryor JA, Morrison JC. Abdominal pregnancy: Current concepts of management. Obstet Gynecol. 1988; 71:549-57.

7. Naim NM, Ahmad S, Siraj HH, Ng P, Mahdy ZA, Razi ZR. Advanced abdominal pregnancy resulting from late uterine rupture. Obstet Gynecol. 2008;111(2 Pt 2):502-4.

8. Bayless RB. Nontubal ectopic pregnancy. Clin Obstet Gynecol. 1987; 30:191-9.

9. Little KJ, Green MM. Abdominal gestation. J Emerg Med. 1995;13:195-8.

10. Ali V, Saldana LR, BaIat IY, Katragadda R. Pitfalls in sonographic diagnosis of abdominal pregnancy. South Med J.1981;74:477-9.

11. Audifred JR, Herrera A, Gonzalez MG, Estrada SF. Embarazo ectópico intraligamentario. Ginecol Obstet Mex. 2013;81:211-4.

12. Mahi M, Boumdin H, Chaouir S, Salaheddine T, Attioui D, Amil T, et al. A new case of abdominal pregnancy. J Radiol. 2002;83:989-92.

13. Oneko O, Petru E, Masenga G, Ulrich D, Obure J, Zeck W. Management of the placenta in advanced 
abdominal pregnancies at an East african tertiary referral center. J Womens Health (Larchmt). 2010;19:1369-75.

14. Harirah HM, Smith JM, Dixon CL, Hankins GD. Conservative management and planned surgery for periviable advanced extrauterine abdominal pregnancy with favorable outcome: Report of two cases. AJP Rep. 2016;6:e301-8.

15. Udigwe GO, Eleje GU, Ihekwoaba EC, Udegbunam OI, Egeonu RO, Okwuosa AO. Acute intestinal obstruction complicating abdominal pregnancy: Conservative management and successful outcome. Case Rep Obstet Gynecol. 2016;2016:2576280.

16. Nassali MN, Benti TM, Bandani-Ntsabele M, Musinguzi E. A case report of an asymptomatic late term abdominal pregnancy with a live birth at 41 weeks of gestation. BMC Res Notes. 2016;9:31.

17. El-Agwany AS, El-Badawy El-S, El-Habashy A, El-Gammal H, Abdelnaby M. Secondary advanced abdominal pregnancy after suspected ruptured cornual pregnancy with good maternal outcome: A case with unusual gangrenous fetal toes and ultrasound diagnoses managed by hysterectomy. Clin Med Insights Womens Health. 2016;9:1-5.

18. Bhoil R, Aggarwal N, Jhobta A, Sharma S. Advanced abdominal pregnancy with successful outcome. Intern Emerg Med. 2016;11:877-8.

19. Brouard KJ, Howard BR, Dyer RA. Hepatic Pregnancy Suspected at Term and Successful Delivery of a Live Neonate With Placental Attachment to the Right Lobe of the Liver. Obstet Gynecol. 2015;126:207-10.

20. Mengistu Z, Getachew A, Adefris M. Term abdominal pregnancy: A case report. J Med Case Rep. 2015;9:168.

21. Kim MC, Manyanga H, Lwakatare F. Secondary abdominal pregnancy in human immunodeficiency virus-positive woman. Obstet Gynecol Sci. 2016;59:535-8.

22. Gidiri MF, Kanyenze M. Advanced abdominal ectopic pregnancy: Lessons from three cases from Zimbabwe and a literature appraisal of diagnostic and management challenges. Womens Health. 2015;11:275-9.

23. Lee C. Abdominal pregnancy in a low-resource setting. Obstet Gynecol. 2015;125:1039-41.

24. Bohiltea R, Radoi V, Tufan C, Horhoianu IA, Bohiltea C. Abdominal pregnancy - Case presentation. J Med Life. 2015;8:49-54.
25. Dabiri T, Marroquin GA, Bendek B, Agamasu E, Mikhail M. Advanced extrauterine pregnancy at 33 weeks with a healthy newborn. Biomed Res Int. 2014;2014:102479. doi: 10.1155/2014/102479.

26. Fouelifack FY, Fouogue JT, Fouedjio JH, Sando Z. Viable abdominal pregnancy: A case report in Yaoundé (Cameroon). Pan Afr Med J. 2014;18:181.

27. Huang K, Song L, Wang L, Gao Z, Meng Y, Lu Y. Advanced abdominal pregnancy: An increasingly challenging clinical concern for obstetricians. Int J Clin Exp Pathol. 2014;7:5461-72.

28. Marcellin L, Ménard S, Lamau MC, Mignon A, Aubelle MS, Grangé G, et al. Conservative management of an advanced abdominal pregnancy at 22 weeks. AJP Rep. 2014;4:55-60.

29. Joshi B, Aggarwal N, Singh A. Obstetrical catastrophe averted: Successful outcome of an abdominal pregnancy. Am J Emerg Med. 2014;32:1299.

30. Matovelo D, Ng'walida N. Hemoperitoneum in advanced abdominal pregnancy with a live baby: A case report. BMC Res Notes. 2014;7:106.

31. Renfroe S, Dajani NK, Pandey T, Magann EF. Role of serial MRI assessment in the management of an abdominal pregnancy. BMJ Case Rep. 2013;2013: bcr2013200495.

32. Smrtka MP, Gunatilake R, Miller MJ, Heine RP, Brown HL. Improving the management of an advanced extrauterine pregnancy using pelvic arteriography in a hybrid operating suite. AJP Rep. 2012;2:63-6.

33. Aliyu LD, Ashimi AO. A multicentre study of advanced abdominal pregnancy: A review of six cases in low resource settings. Eur J Obstet Gynecol Reprod Biol. 2013;170:33-8.

34. Mpogoro F, Gumodoka B, Kihunrwa A, Massinde A. Managing a live advanced abdominal twin pregnancy. Ann Med Health Sci Res. 2013;3:113-5.

35. Pradhan M, Sreenivas M, Singh B, Behera C, Dikshit PC. Sudden death in advanced abdominal pregnancy: A case report and discussion of the related medicolegal issues. Med Sci Law. 2013;53:90-2.

36. Masukume G, Sengurayi E, Muchara A, Mucheni E, Ndebele W, Ngwenya S. Full-term abdominal extrauterine pregnancy complicated by post-operative ascites with successful outcome: A case report. J Med Case Rep. 2013;7:10.

37. Kim MJ, Bae JY, Seong WJ, Lee YS. Sonographic diagnosis of a viable abdominal pregnancy with 
planned delivery after fetal lung maturation. J Clin Ultrasound. 2013;41:563-5.

38. Mittal SK, Singh N, Verman AK, Agarwal H, Kulkarni CD, Kanaujia R. Fetal MRI in the pre-operative diagnosis and assessment of secondary abdominal pregnancy: A rare sequela of a previous caesarean section. Diagn Interv Radiol. 2012;18:496-502.

39. Collins SL, Grant D, Black RS, Vellayan M, Impey L. Abdominal pregnancy: A perfusion confusion? Placenta. 2011;32:793-5.

40. Dahab AA, Aburass R, Shakkat W, Babgi R, Essa O, Mujallid RH. Full term extrauterine abdominal pregnancy: A case report. J Med Case Rep. 2011;5:531.

41. Baffoe P, Fofie C, Gandau BN. Term abdominal pregnancy with healthy newborn: A case report. Ghana Med J. 2011;45:81-3.

42. Brewster EM Sr, Braithwaite EA, Brewster EM Jr. Advanced abdominal pregnancy: A case report of good maternal and perinatal outcome. West Indian Med J. 2011;60:587-9.

43. Ramphal SR, Moodley J, Rajaruthnam D. Hepatic pregnancy managed conservatively. Trop Doct. 2010; 40:121-2.

44. Gupta P, Sehgal A, Huria A, Mehra R. Secondary abdominal pregnancy and ir associated diagnostic and operative dilemma: Three case reports. J Med Case Rep. 2009;3:7382.

45. Sunday-adeoye I, Twomey D, Egwuatu EV, Okonta PI. A 30 year review of advanced abdominal pregnancy at the mater misericordiae hospital, a fikpo, southeastern Nigeria (1976-2006). Arch Gynecol Obstet. 2011;283:19-24.
46. Worley KC, Hnat MD, Cunningham FG. Advanced extrauterine pregnancy: Diagnostic and therapeutic challenges. Am J Obstet Gynecol. 2008;198:297.e1-7.

47. Isah AY, Ahmed Y, Nwobodo EI, Ekele BA. Abdominal pregnancy with a full term live fetus: Case report. Ann Afr Med. 2008;7:198-9.

48. Promsonthi P, Herabutya Y. Uterocutaneous fistula in term abdominal pregnancy. Eur J Obstet Gynecol Reprod Biol. 2007;132:239-41.

49. Lockhat F, Corr P, Ramphal S, Moodley J. The value of magnetic resonance imaging in the diagnosis and management of extra-uterine abdominal pregnancy. Clin Radiol. 2006;61:264-9.

50. Roberts RV, DickinsonI JE, Leung Y, Charles AK. Advanced abdominal pregnancy: Still an occurrence in modern medicine. Aust N Z J Obstet Gynaecol. 2005;45:518-21.

51. Tshivhula F, Hall DR. Expectant management of an advanced abdominal pregnancy. J Obstet Gynaecol. 2005;25:298.

52. Godyn JJ, Hazra A, Gulli VM. Subperitoneal placenta accreta succenturiate in the case of a successful nearterm extrauterine abdominal pregnancy. Hum Pathol. 2005:36:922-6.

53. Cetinkaya MB, Kokcu A, Alper T. Follow up of the regression of the placenta left in situ in an advanced abdominal pregnancy using the Cavalieri method. J Obstet Gynaecol Res. 2005;31:22-6.

54. Xiao GH, Chen DJ, Sun XF, She RQ, Mai YM. Abdominal pregnancy: Full-term viable baby. Eur J Obstet Gynecol Reprod Biol. 2005;118:117-8. 